\title{
Bacillus pumilus
}

National Cancer Institute

\section{Source}

National Cancer Institute. Bacillus pumilus. NCI Thesaurus. Code C122181.

A species of a Gram-positive, aerobic, spore-forming bacillus in the family Bacillaceae.

The species is positive for protease and lipase but negative for amylase, phosphatase,

DNase, gelatinase and chitinase. B. pumilus is commonly found in soil and aquatic environments. 